\title{
Erfahrungsaustausch mit den kommunalen Datenschutzbeauftragten
}

Datenschutz ist Grundrechtsschutz und zum Schutz der Grundrechte sind a lle in Staat und Gesellschaft berufen. Hierfür ist ein Mindestmaß an Koordination und Kooperation nötig. Beim Datenschutz obliegt das eine wie das andere den Datenschutzbeauftragten des Bundes und der Länder. In Rheinland-Pfalz habe ich mir deshalb die Kooperation mit den behördlichen und betrieblichen Datenschutzbeauftragten zu einer meiner Hauptaufgaben gemacht.

Sie beinhaltet vor allem den Aufbau entsprechender Netzwerke. Die ersten Schritte dazu sind bereits getan. Mit Hilfe des hiesigen Schulministeriums verfügt der LfD über die E-Mail-Adressen nahezu aller schulischen Datenschutzbeauftragten. Auf diese Weise werden rund 1200 schulische Datenschutzbeauftragte über aktuelle datenschutzrechtliche Entwicklungen, vor allem im Schulbereich informiert und zu entsprechenden Veranstaltungen des LfD eingeladen.

Ähnliches geschieht mit den behördlichen Datenschutzbeauftragten in den obersten Landesbehörden und in der Justizverwaltung.

Im Mittelpunkt unserer jüngsten Aktivitäten stehen die behördlichen Datenschutzbeauftragten in den Kommunen. Bereits im vergangenen Jahr hatten wir die behördlichen Datenschutzbeauftragten der Landkreise, kreisfreien und großen kreisangehörigen Städte zu einem ersten Treffen eingeladen. Die Veranstaltung mit über 30 Datenschutzbeauftragten war die erste dieser Art, seitdem 1994 durch das LDSG behördliche Datenschutzbeauftragte in Rheinland-Pfalz eingeführt worden waren.

Eine Anregung dazu erhielt ich auch durch einen Hinweis auf der Homepage des Landesbeauftragten für den Datenschutz Niedersachsen auf das „Netzwerk NORDWEST“, in dem sich 2005 mit Unterstützung des niedersächsischen LfD kommunale Datenschutzbeauftragte zur gegenseitigen Unterstützung zusammengeschlossen haben. Die treibenden Kräfte dieses Netzwerks waren der Datenschutzbeauftragte des Landkreises Diepholz sowie der damalige Datenschutzbeauftragte der Stadt Oldenburg. Sie hatte ich zu diesem ersten Treffen eingeladen, damit sie von ihren praktischen Erfahrungen berichten.

Um die Bemühungen für eine stärkere Zusammenarbeit meiner Dienststelle mit den kommunalen Datenschutzbeauftragten $\mathrm{zu}$ verstetigen und den Aufbau eines solchen Netzwerkes in Rheinland-Pfalz voranzutreiben, fand am 20. Oktober 2008 ein zweiter Gedankenaustausch statt, zu dem erstmals auch die behördlichen Datenschutzbeauftragten der 192 Verbandsgemeinden sowie verbandsfreien Gemeinden und Städte eingeladen wurden. Begrüßen konnte ich aus den 236 Kommunen dann knapp 100 Datenschutzbeauftragte - eine beachtliche Zahl!

Im Rahmen der Veranstaltung wurden unter dem Stichwort „Selbstverständnis der kommunalen Datenschutzbeauftragten" die Ergebnisse einer Umfrage unter den behördlichen Datenschutzbeauftragten der Landkreise, kreisfreien und großen kreisangehörigen Städten dargestellt. Ein Resultat war überraschend: ein Anteil von 5\% der Arbeitszeit reicht den behördlichen Datenschutzbeauftragten offenbar für die Ausübung ihrer Tätigkeit aus.

Ein weiteres Ergebnis war, dass das Internet-Angebot des Landesdatenschutzbeauftragten nach dem Wunsch der Teilnehmer verstärkt auf Fragen und Probleme dieser Zielgruppe eingehen soll.

In der Sitzung wurden auch verschiedene Sachfragen thematisiert. Die Palette reichte von Fragen der richtigen Gestaltung von Anbieterkennzeichnungen im Internet über die Melderegisterauskünfte an Privatunternehmen bis zum Einsatz von Videokameras und Webcams durch Gemeinden. In diesem Zusammenhang bestand für mich die Gelegenheit, für ei- nen zurückhaltenden Einsatz dieser Technik zu werben. Das zweite Treffen bot die Möglichkeit, in zahlreichen Gesprächen Hemmschwellen untereinander sowie im Verhältnis zu mir und den Mitarbeiterinnen und Mitarbeitern meiner Dienststelle abzubauen. Weiterhin konnten die Kommunalen Spitzenverbände „ins Boot“ geholt und für die Idee eines „Datenschutznetzwerkes" unter den kommunalen Datenschutzbeauftragten gewonnen werden. Nicht unerwähnt bleiben soll auch, dass der Gedankenaustausch sowohl für die Tageszeitungen als auch den SWR von Interesse war und das Thema "Datenschutz“ auch in diesem Zusammenhang präsent war.

Fazit

Es ist anzustreben, das Engagement der kommunalen Datenschutzbeauftragten für ihre Tätigkeit und auch ihre Mitarbeit bei solchen Treffen zu verbessern. Der gewählte Personenkreis war für eine aktive und fruchtbare Mitarbeit vielleicht $\mathrm{zu}$ groß. Möglicherweise ist es zielführender, Treffen nur mit den Datenschutzbeauftragten der Landkreise, kreisfreien und großen kreisangehörigen Städten oder getrennt nach Regionen durchzuführen. Die Überlegungen in meiner Dienststelle sind dazu noch nicht abgeschlossen. Sicherlich wird ein „langer Atem“ notwendig sein, bis ein solches Netzwerk funktioniert und jeder vom Wissen und Können der anderen profitiert. Ich halte ein Netzwerk aber schon deshalb für sinnvoll, um einen relativ großen Personenkreis vor Ort unmittelbar und zeitnah mit datenschutzrechtlichen Anliegen ansprechen und damit zur Stärkung und Verbesserung des Datenschutzes beitragen zu können.
Edgar Wagner
Landesbeauftragter für den Datenschutz Rheinland-Pfalz 